\title{
Véhicules coopératifs pour une gestion dynamique du trafic : approche théorique et simulation
}

\author{
Connected vehicles for Intelligent Transportation systems: theoretical approach and simulation
}

\author{
Julien Monteil · Romain Billot · Nour-Eddin El Faouzi
}

Reçu le 18 mai 2012 ; accepté le 20 décembre 2012

(C) IFSTTAR et Éditions NecPlus 2013

Résumé Les avancées en technologies de la communication ont peu à peu donné naissance à une deuxième génération de Systèmes de transports intelligents (ITS) connus sous le nom de systèmes coopératifs. En permettant aux différents véhicules de communiquer entre eux ou à travers l'infrastructure, les systèmes coopératifs révolutionnent la gestion du trafic autant qu'ils la complexifient de par la richesse des possibilités qu'ils entrouvrent. L'implémentation prochaine de ces nouveaux services pose également un certain nombre de défis en modélisation du trafic qu'il convient d'anticiper. Dans cet article, nous proposons un effort d'intégration de quelques concepts de communication dans les modèles microscopiques de type loi de poursuite et les stratégies de changement de voie associées. Au niveau théorique, les analyses de stabilité montrent comment l'introduction d'une coopération permet, dans certaines situations, de limiter le développement d'ondes de type stop and go. Ces résultats sont ensuite illustrés en

Remerciements Ces travaux ont été en partie réalisés dans le cadre du réseau d'excellence Nearctis (www.nearctis.org) et du projet Co-Drive.

Julien Monteil $(\triangle)$

Université de Lyon, F-69000, Lyon, France

IFSTTAR, COSYS/LICIT, F-69675, Bron

ENTPE, LICIT, F-69518 Vaulx-en-Velin

25 av. François Mitterrand, Cité des mobilités,

Case 24, F-69675 Bron cedex

e-mail : julien.monteil@ifsttar.fr

Romain Billot

Université de Lyon, F-69000 Lyon, France

IFSTTAR, COSYS/LICIT, F-69675, Bron

ENTPE, LICIT, F-69518 Vaulx-en-Velin

25 av. François Mitterrand, Cité des mobilités, Case 24, F-69675

Bron cedex

e-mail : romain.billot@entpe.fr

Nour-Eddin El Faouzi

Université de Lyon, F-69000 Lyon, France

IFSTTAR, COSYS/LICIT, F-69675, Bron

ENTPE, LICIT, F-69518 Vaulx-en-Velin

25 av. François Mitterrand, Cité des mobilités, Case 24, F-69675

Bron cedex

e-mail : nour-eddin.elfaouzi@ifsttar.fr simulation à travers le calibrage de données réelles. Les expériences numériques permettent ainsi de conclure sur le potentiel stabilisant des systèmes coopératifs en situation de congestion. Des considérations sur le pourcentage de véhicules ou le rayon de communication permettent de fournir des premiers éléments d'aide à la décision en vue de futurs déploiements de ces nouvelles technologies.

Mots clés véhicules coopératifs · communication intervéhiculaire · modélisation du trafic · stabilité linéaire

\begin{abstract}
The expected future deployment of communication technologies, and the new generation of Intelligent Transportation Systems (ITS) known as cooperative systems bring lots of expectations in terms of vehicle traffic flow control and management. The numbers of interactions and exchanges between cars, and with the infrastructure can considerably grow, which as a result could carry new challenges to the traffic modeling community. In this paper we investigate the effects of cooperation between vehicles at a microscopic level, where communication is directly integrated into the car following law. Some linear stability analyses allow identifying regimes of potential propagation of shock waves into a traffic stream. Real data based simulation confirms the theoretical findings, and values of efficiency and safety indicators are discussed based on the chosen cooperative strategy.
\end{abstract}

Keywords connected vehicles - vehicle to vehicle communication $\cdot$ traffic modelling $\cdot$ linear stability analysis

\section{Introduction}

\section{Qu'est ce qu'un système coopératif ?}

La gestion du trafic s'est longtemps appuyée sur des données trafic de type boucles électromagnétiques, recueillies à partir de postes de comptages fixes. Ces données permettent le comptage des véhicules, la mesure de leur vitesse et l'estimation de la densité locale du trafic. Ces installations 


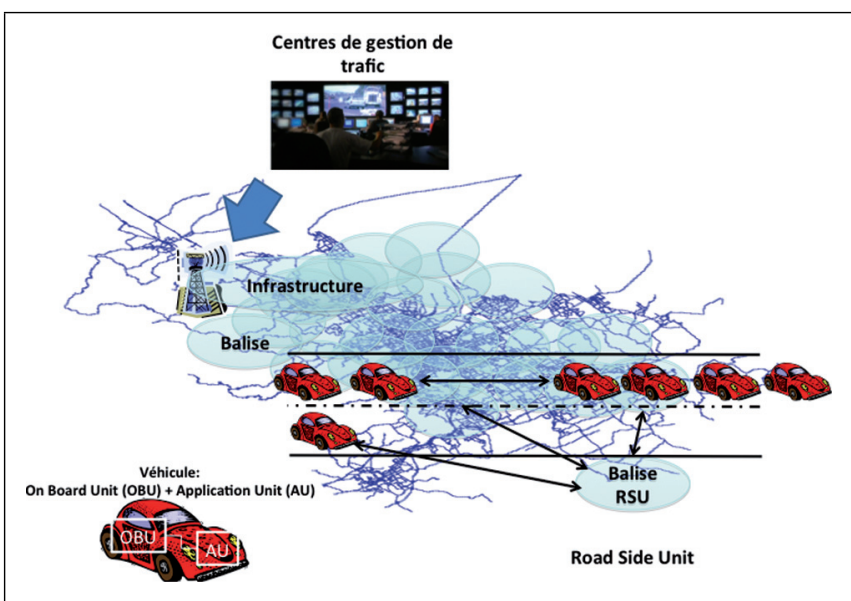

Fig. 1 Architecture de communication

coûteuses ont très vite contraint les gestionnaires à limiter leurs efforts à des zones d'intérêt ou à risque (ex : zone accidentelle ou étude en vue de l'installation d'un radar). Les années 1990 auront vu le déploiement des systèmes de transports intelligents (ITS) et l'utilisation des technologies de l'information et de la communication comme autant d'outils d'aide à la gestion du trafic. Les données temporelles en un point fixe ont ainsi été complétées par des données trajectoires qui suivent des véhicules sur une certaine zone d'étude. Il s'agit des données trajectoires provenant de véhicules équipés FCD (Floating Car Data) ou de traces GPS. C'est ainsi que les véhicules sont devenus eux-mêmes des capteurs plutôt que de simples entités captées. La décennie précédente aura été également et surtout celle de l'équipement du véhicule et de la multiplication des capteurs intra-véhiculaires. Les systèmes d'aide à la conduite renseignent le conducteur sur la stabilité de son véhicule ou sur les aspects latéraux et longitudinaux. En sus de conditions météorologiques dégradées, comme une visibilité faible, les véhicules sont maintenant capables de détecter des obstacles ou des zones d'adhérence réduite. Après cette informatisation du véhicule par rapport à son environnement extérieur, il y a fort à parier que la décennie prochaine sera celle de la connexion de l'infrastructure aux véhicules et des véhicules entre eux. On appelle systèmes coopératifs l'ensemble des outils d'aide à la conduite ou la gestion fondé sur les technologies de la communication. Ces systèmes sont maintenant considérés comme la deuxième génération des systèmes ITS. Les technologies considérées sont les communications C2C (car to car), C2I (car to infrastructure) et I2C (infrastructure to car). Les acteurs du système de communication coopérative sont les conducteurs qui reçoivent des informations et autres recommandations, les exploitants du réseau (qui reçoivent et centralisent les données de trafic et peuvent renvoyer des consignes de contrôle du trafic) et potentiellement les services internet (fournisseurs d'accès à travers les hotspots). La figure 1 illustre les différents types d'échanges et ainsi l'architecture de communication d'un réseau coopératif.

Un véhicule dit coopératif est composé d'une unité de bord ou on-board unit (OBU) et d'une unité d'application ou application unit (AU) (figure 1). L'AU est le dispositif qui exécute les applications et tire profit des capacités de communication de l'OBU. L'AU et l'OBU peuvent être reliées par connexion sans fil (bluetooth) ou filaire.

Le réseau ad hoc ou Vehicular Ad Hoc Network (Vanet) est composé de véhicules équipés avec des OBU et des unités stationnaires le long de la route, appelées balises ou road side units (RSU). Les OBU forment entre elles un réseau mobile ad hoc (ou Manet, Mobile Ad hoc NETwork) qui permet la communication d'une manière complètement distribuée sans aucune instance de centralisation.

Le paradigme coopératif repose sur la croyance qu'une multiplication des échanges entre véhicules, conducteurs ou infrastructure ira de pair avec une amélioration des conditions de circulation. Cette confiance en ces nouvelles technologies se traduit en un certain nombre de projets industriels ou d'initiatives de recherche qui sont détaillés par la suite.

\section{Contexte et enjeux}

L'expansion des systèmes coopératifs semble aujourd'hui bénéficier d'un contexte européen favorable. Au sein du cadre d'harmonisation Européen EasyWay ${ }^{1}$, un groupe d'experts pilote des actions ciblées sur les systèmes coopératifs et la nature des stratégies de gestion à mettre en place en priorité (alertes de congestion, notification d'un danger, guidage temps-réel). Au niveau des systèmes d'aide à la conduite, un certain nombre de projets de recherche ont permis d'analyser les concepts et d'élaborer des solutions technologiques ouvrant la voie à une phase d'industrialisation. Quant aux systèmes coopératifs à proprement parler, ils sont le corps de nombreux projets de recherche actuels. Au niveau européen, on peut citer CVIS $^{2}$ (systèmes coopératifs avec orientation vers la gestion de trafic), $\mathrm{SafeSpot}^{3}$ (marges de sécurité des véhicules), Coopers $^{4}$ (technologies de télécommunication pour les autoroutes) ou Drive. Au niveau français, citons Predit 99, Arcos et Plata ou Modurban. Plus récemment, le projet Co-Drive (Copilote pour une route intelligente et des véhicules communicants) vise à développer un système qui offrira un outil complémentaire de gestion dynamique des infrastructures et du trafic par la remontée et le traitement de données (véhicules $\&$ environnement routier) et la diffusion de consignes aux usagers (vitesses réglementaires, distance

\footnotetext{
${ }^{1} \mathrm{http}: / /$ www.easyway-its.eu/

$2 \mathrm{http}: / /$ www.cvisproject.org/

${ }^{3} \mathrm{http}: / /$ www.safespot-eu.org/

${ }^{4} \mathrm{http}: / / \mathrm{www}$. coopers-ip.eu/index.php?id=project
} 
de sécurité entre véhicules, alertes d'événements locaux ou distants).

Au niveau de la communication, une bande de fréquence a été allouée au plan Européen $(5,875-5,905 \mathrm{Ghz})$ pour couvrir les besoins liés à la gestion du trafic et à la sécurité routière. Les informations récoltées sur les trajectoires de véhicules, les vitesses, les changements de voie, la consommation, permettraient ainsi de bâtir des indicateurs de trafic toujours plus performants. Au niveau de la sécurité, le standard $802.11 \mathrm{p}$ (porté par le projet Scoref ${ }^{5}$ ) gère par exemple des canaux de sécurité prioritaires et la norme est admise par un large groupe d'acteurs (Car to car consortium, 2007) et en particulier dans des projets européens comme CVIS ${ }^{6}$ ou GeoNet. De nombreux messages associés aux différents services envisagés sont déjà en cours de normalisation. Ces messages couvrent les informations trafic, les services de paiement, les communications véhicule-infrastructure et véhicule-véhicule. Ils formeront le noyau d'une optimisation multi-objectif du trafic.

Les enjeux sont considérables. Les différents niveaux d'interaction mis en place par les systèmes coopératifs peuvent potentiellement mener à une transformation des comportements des usagers et, de fait, à une nouvelle façon de gérer le trafic. Dans une vision coopérative de la circulation routière, les véhicules échangent entre eux ou avec l'infrastructure une information relative à la section de route traversée. Les données reçues peuvent être traitées en temps réel et renvoyées aux véhicules sur la section considérée, sous forme individuelle ou groupée. En étendant les possibilités offertes par les systèmes autonomes au-delà des simples risques de collision, les systèmes coopératifs permettent au conducteur d'interagir avec son environnement et d'avoir une meilleure perception des conditions de circulation. Par exemple, une information sur les conditions d'adhérence par conditions pluvieuses prendra encore plus d'importance si elle est couplée à des indications sur les conditions de circulation en aval. Ces nouvelles technologies ouvrent la voie à une régulation de vitesse adaptative et coopérative (Cooperative and Adaptative Cruise Control - CACC) [1].

Au niveau de la sécurité routière, l'information en temps réel sur le niveau de risque ne profitera pas seulement à l'usager mais aussi au gestionnaire à travers des délais d'intervention réduits. De plus, le fait d'avoir accès à une gestion facilitée de la vitesse et des inter-distances par une programmation aisée des limiteurs de vitesses ou régulateur de vitesse pourrait, en plus de transmettre en temps réel une information liée au risque de collision en cas de conduite à risque, être également un facteur d'incitation au développement de véhicules électriques. Les

\footnotetext{
$\overline{5}$ wiki.fot-net.eu/index.php?title=SCOREF

${ }^{6} \mathrm{http}: / /$ www.cvisproject.org/
}

gestionnaires de trafic auront un rôle important à jouer dans l'assimilation des différentes sources de données et le contrôle résultant sur les véhicules. À travers la communication infrastructure-véhicule, les actions de régulation de vitesse pourraient être ciblées par catégories de véhicules, des véhicules plus modérés en termes d'émissions de $\mathrm{CO} 2$ (covoiturage, auto-partage, véhicules électriques) étant informés différemment que d'autres. La notion de contrôle d'accès (ramp metering) pourrait également être revisitée à la lumière des possibilités offertes par les systèmes coopératifs. Les véhicules souhaitant s'insérer recevraient une information pertinente de la part des gestionnaires sur la vitesse à adopter ou pourquoi pas une redirection en cas de congestion. D'autre part, en milieu urbain, il semble prometteur de repenser le concept d'ondes vertes, en portant le travail non seulement sur la synchronisation optimale des feux mais aussi et surtout sur les informations à fournir aux véhicules pour fluidifier le trafic et limiter la formation de longues files de véhicules aux intersections.

\section{Limites et besoin en recherche}

Malgré la potentielle richesse des systèmes coopératifs en vue d'une nouvelle régulation dynamique du trafic, il convient de rappeler que les éléments sus-cités se situent pour le moment à un niveau prospectif. Au niveau de la recherche en modélisation du trafic, la passerelle entre systèmes coopératifs et contrôle macroscopique doit être créée en commençant par l'introduction des aspects coopératifs dans les modélisations usuelles du trafic. Cette tâche n'est pas simple dans la mesure où cette recherche en amont ne peut s'appuyer sur aucune source de données coopératives pour calibrer et valider les modèles proposés. La proposition et la simulation de modèles coopératifs constitueraient pourtant une base solide afin de tester des questions ouvertes pour les industriels et décideurs. Parmi les interrogations auxquelles les simulations pourraient répondre, citons par exemple le pourcentage minimum de véhicules équipés (encore connu sous le terme de taux de pénétration) nécessaire à une amélioration des conditions de circulation. Une fraction partielle de véhicules équipés au sein d'un trafic hétérogène pourrait toutefois créer des " interférences » au niveau des comportements de conducteurs, informés ou non, menant à des effets contre-productifs. L'utilisation de la gestion coopérative pourrait ainsi dépendre de la composition du trafic et de son état (densité par exemple). Du point de vue de la communication, les échanges sont limités par le rayon d'interaction et il serait pertinent de quantifier l'impact de cette distance sur l'état du système. Quant à la nature des informations à envoyer, leur réception et utilisation par les conducteurs, et les effets sous-jacents sur le flot de véhicules, c'est assurément un point critique devant susciter 
un effort interdisciplinaire. En vue d'une implémentation prochaine, les systèmes coopératifs doivent ainsi faire l'objet d'un certain nombre de recherches aux niveaux théorique et appliqué.

\section{Objectifs et organisation de l'article}

Notre travail se veut un effort de recherche théorique visant à mieux comprendre et caractériser l'impact potentiel des systèmes coopératifs sur le trafic routier, en particulier dans des conditions de forte densité de trafic ou des situations de congestion. Nous proposons une première approche d'intégration des principes coopératifs dans les modèles de trafic à travers le développement et l'analyse de modèles de trafic multi-anticipatifs (section 2). Nous analysons ensuite les bénéfices apportés par les systèmes coopératifs à travers le concept de stabilisation du trafic. Des résultats de stabilité linéaire sont proposés pour des classes génériques de modèles microscopiques. Ces analyses sont étendues dans le domaine non linéaire, les conclusions apportant des éléments sur la structure des ondes de choc formées (section 3). Dans une quatrième section, des résultats de simulation sont présentés en utilisant un modèle microscopique précis. Le modèle est calibré à partir de données réelles de trajectoires et les résultats confirment les analyses théoriques. Les changements de voie sont introduits, qui permettent de considérer l'agressivité des conducteurs en parallèle des aspects coopératifs. Les impacts du taux de pénétration et du rayon de communication sont présentés en observant des indicateurs de trafic ou de sécurité bien connus des lecteurs. L'article est conclu par une discussion sur les limites de l'étude et une ouverture sur les pistes de recherches foisonnantes autour de la thématique des systèmes coopératifs (section 5).

\section{Comment intégrer la coopération dans la modélisation du trafic?}

Si les modèles de trafic forment le cœur de la plupart des outils d'aide à la décision destinés aux gestionnaires, il faut remarquer qu'ils n'ont pas été développés pour prendre en compte toutes les interactions possibles entre des entités communicantes sur le réseau. La problématique est ambivalente puisqu'il s'agit d'un côté d'analyser l'impact des systèmes coopératifs sur les différents paramètres pour mieux calibrer les modèles (ex : vitesses ou temps inter-véhiculaire) et d'un autre côté de proposer dans un futur proche un cadre de modélisation nouveau pour mieux traduire la communication entre les différents éléments du réseau. Ces deux blocs de recherche interagiront forcément dans la mesure où les modèles de trafic classiques (modèles microscopiques de poursuite ou automates cellulaires, modèles macroscopiques du $1^{\mathrm{er}}$ ou $2^{\mathrm{e}}$ ordre) pourraient être partie intégrante d'un nouveau cadre de modélisation, qui consisterait à intégrer une couche d'abstraction supplémentaire pour la prise en compte de l'aspect communicatif. Une fois validée, cette modélisation coopérative du trafic permettra d'évaluer a priori les nouvelles solutions proposées, et de tester l'effet de déploiement de systèmes coopératifs sur le trafic en fonction de leur taux de pénétration. Mais avant une telle étape, il convient de déterminer dans quelle mesure les principes coopératifs peuvent être introduits dans les formalismes de modélisation actuels. Après un état de l'art sur le sujet, nous proposons un cadre d'intégration d'une communication inter-véhiculaire reposant sur une extension multi-anticipative des modèles microscopiques.

\section{État de l'art}

Alors que l'on dénote un certain nombre de travaux portant sur la route automatique ou le platooning, la modélisation des systèmes coopératifs et surtout la communication inter-véhiculaire retient l'attention de la communauté scientifique depuis une période plus récente. L'approche naturelle consiste à étendre les modèles existants afin d'introduire de la communication. Ngoduy et al. divisent le trafic entre deux classes de véhicules équipés et non équipés. Les véhicules équipés sont capables de recevoir de l'information sur les conditions de circulation en aval. Cette information permet d'anticiper la formation de la congestion et d'adopter une phase de décélération vers une vitesse de bas régime. Pour ce faire, un modèle cinétique multi-classe est utilisé avec deux classes de véhicules. Une fonction représentant l'information traduit la probabilité qu'un véhicule équipé reçoive un message d'un véhicule en aval. Les résultats numériques montrent que la présence de véhicules équipés contribue à une stabilisation du trafic (en termes de quantité et amplitude des ondes) et à une baisse des temps de parcours. En raison de l'absence de données coopératives réelles, les résultats n'ont pu être validés sur des données réelles. Les contributions au niveau macroscopique sont rares car il apparaît plus cohérent de commencer au niveau microscopique de la communication entre véhicules ou avec l'infrastructure. Une démarche de type bottom-up pourra ainsi faire émerger, à partir d'une modélisation microscopique, des propriétés résultantes au niveau macroscopique du flot. Au niveau microscopique, les efforts actuels consistent à étendre les modèles de type loi de poursuite afin d'y intégrer la communication entre véhicules. Ces modèles reposent sur l'idée qu'un véhicule met à jour sa vitesse en fonction de sa perception de l'inter-distance et de la vitesse du véhicule précédent [2].

Les comportements de modèles largement utilisés comme le modèle Optimal Velocity (OV) de Bando, le generalized force model ou intelligent driver model de Helbing et Treiber sont reconnus comme reproduisant de façon 
satisfaisante la dynamique du trafic en conditions critiques. En ce qui concerne la communication entre véhicules, quelques extensions de ces modèles ont été proches des concepts coopératifs. Ainsi, Ge et al. étendent les modèles classiques de telle sorte qu'un véhicule adapte sa vitesse ou son accélération en fonction de multiples véhicules situés devant lui. Les auteurs montrent que le cadre multianticipatif permet d'améliorer la stabilité du trafic. Dans la suite, nous généralisons cette démarche et introduisons de nouveaux résultats de stabilité. Notre effort de recherche approfondit ainsi les études existantes au niveau théorique, tout en étant inédit dans la considération des systèmes coopératifs au niveau microscopique [3] [4] [5] [6].

Notons que de telles analyses de stabilité renvoient parfois à des travaux plus anciens portant sur les routes automatiques. Il s'agissait alors d'étudier le développement d'oscillations parmi un groupe de véhicules suivant un leader à vitesses fixes. Nous renvoyons le lecteur aux travaux de Ioannou et Bose pour une bonne introduction à la notion de string instability. ou au projet California Path sur le thème de la route automatique. D'autre part, une classification mathématique des analyses de stabilité est proposée dans Treiber et Kesting [7] [8].

\section{Une première approche : la coopération par la multi-anticipation au niveau microscopique}

Dans un environnement coopératif, le véhicule ajuste sa vitesse et son accélération en fonction de plusieurs véhicules situés à l'aval. Nous proposons une approche multi-anticipative dans laquelle un véhicule adapte à chaque pas de temps son accélération selon l'information située dans un certain rayon d'interaction. Aucune source de bruit telle qu'un temps de latence dans la communication ou une éventuelle défaillance n'est, à ce stade, introduite. On note $r$ le rayon d'interaction entre véhicules, de telle sorte que les voisins informatifs d'un véhicule i sont définis comme

$$
N_{i}=\left\{j \in V: 0 \leq x_{j}-x_{i} \leq r\right\}
$$

où $\mathrm{x}_{\mathrm{j}}$ représente la position du véhicule $\mathrm{i}$, et $|\mathrm{V}|$ le nombre total de véhicules dans la section étudiée. Ceci définit un réseau de proximité, qui est la topologie d'un réseau de véhicules capteurs sans fil dans un rayon $r$. Ce rayon est généralement de l'ordre de 250 mètres. L'accélération d'un véhicule $n$ est notée $\ddot{x}_{n}$, sa vitesse $\dot{x}_{n}$ tandis que $\Delta x_{n}$ et $\Delta \dot{x}_{n}$ représentent respectivement l'inter-distance et la vitesse relative entre deux véhicules $n$ et $n+1$. En environnement coopératif et multi-anticipatif, l'accélération d'un véhicule est notée

$\ddot{x}_{\mathrm{n}}=\mathrm{f}\left(\dot{\mathrm{x}}_{\mathrm{n}}, \Delta \mathrm{x}_{\mathrm{n}}, \Delta \mathrm{x}_{\mathrm{n}+1} \ldots, \Delta \mathrm{x}_{\mathrm{n}+\mathrm{m}}, \Delta \mathrm{v}_{\mathrm{n}}, \Delta \mathrm{v}_{\mathrm{n}+1} \ldots, \Delta \mathrm{v}_{\mathrm{n}+\mathrm{m}}\right)$ où $\mathrm{m}+1=\left|\mathrm{N}_{\mathrm{n}}\right|$ constitue le nombre de véhicules dans le rayon d'interaction du véhicule (seulement en aval). Afin de complexifier la capitalisation de l'information pour un véhicule nous introduisons des coefficients pondérateurs $a_{j}$ et $b_{j}$ qui amènent à réécrire le modèle de la façon suivante :

$$
\ddot{x}_{\mathrm{n}}=\mathrm{f}\left(\dot{\mathrm{x}}_{\mathrm{n}}, \Sigma_{\mathrm{i}=0}^{\mathrm{m}} \mathrm{a}_{\mathrm{i}} \Delta \mathrm{x}_{\mathrm{n}+\mathrm{i}}, \Sigma_{\mathrm{i}=0}^{\mathrm{m}} \mathrm{b}_{\mathrm{i}} \Delta \dot{\mathrm{x}}_{\mathrm{n}+\mathrm{i}}\right)
$$

$\left(a_{i}\right)_{0 \leq i \leq m}$ et $\left(b_{i}\right)_{0 \leq i \leq m}$ ont des coefficients de proximité qui pondèrent l'interaction entre un véhicule et ses prédécesseurs (numéros $\mathrm{i}+1$ à $\mathrm{m}+1$ ) dans son rayon d'interaction. Ainsi, ces poids traduisent l'importance de chaque véhicule dans la loi coopérative. Plusieurs stratégies de pondération peuvent être proposées et l'élaboration d'une stratégie optimale pourrait bien sûr faire l'objet d'une étude plus poussée. Dans cet article, nous choisissons le cas $\left(b_{\mathrm{i}}\right)_{0 \leq \mathrm{i} \leq \mathrm{m}}=\left(\mathrm{a}_{\mathrm{i}}\right)_{0 \leq \mathrm{i} \leq \mathrm{m}}$ qui met ainsi au même niveau d'intérêt inter-distance et vitesse relative. Les poids sont normalisés de telle sorte que $\Sigma_{\mathrm{i}=0}^{\mathrm{m}} \mathrm{a}_{\mathrm{i}}=1$. Notons aussi que ces coefficients ne dépendent pas du véhicule $\mathrm{n}$ car ils sont choisis en fonction des conditions d'équilibre et non pas des conditions dynamiques de trafic. Cette loi multi-anticipative est en continuité avec la loi sans coopération, qui équivaut à $\mathrm{a}_{0}=1$ et tous les autres coefficients nuls, et qui s'écrit alors :

$$
\ddot{\mathrm{x}}_{\mathrm{n}}=\mathrm{f}\left(\dot{\mathrm{x}}_{\mathrm{n}}, \Delta \mathrm{x}_{\mathrm{n}}, \Delta \dot{\mathrm{x}}_{\mathrm{n}}\right)
$$

Par la suite, la section 3 présente des résultats analytiques concernant les conditions de stabilité du système dynamique (3). Les conditions de stabilité déterminent quand une perturbation se propage ou s'atténue en fonction du temps. Dans le cas d'une amplification de cette perturbation au cours du temps, l'étape suivante est de déterminer l'amplitude de l'onde de choc près de la frontière de stabilité. En effet, il a été observé et prouvé que ce phénomène de perturbation est à l'origine du phénomène stop and go, causant ainsi une chute de capacité. De tels phénomènes apparaissent en cas de freinage brusque ou de changement de voie agressif (insertion, manœuvre pour rejoindre sa voie de destination, ou simple volonté d'aller dans une voie plus rapide). Ces types de manœuvres effectuées dans des conditions de trafic dites instables provoquent une dégradation du trafic [9].

\section{Coopération et stabilité : approche analytique}

L'analyse de stabilité linéaire consiste à étudier l'évolution d'une perturbation autour d'un état d'équilibre. Pour le cas général, un tel état est atteint si

$$
\mathrm{f}\left(\dot{\mathrm{x}}_{\mathrm{eq}}, \Delta \mathrm{x}_{\mathrm{eq}}, 0\right)=0 \text {, }
$$


où $\dot{\mathrm{x}}_{\mathrm{eq}}$ et $\Delta \mathrm{x}_{\mathrm{eq}}$ sont les vitesses et inter-distances d'équilibre. $\mathrm{y}_{\mathrm{n}}$ est la perturbation autour de l'équilibre de telle sorte que :

$$
\begin{gathered}
\Delta \mathrm{x}_{\mathrm{n}}=\Delta \mathrm{x}_{\mathrm{eq}}+\Delta \mathrm{y}_{\mathrm{n}}, \\
\dot{\mathrm{x}}_{\mathrm{n}}=\dot{\mathrm{x}}_{\mathrm{eq}}+\dot{\mathrm{y}}_{\mathrm{n}},
\end{gathered}
$$

avec :

$$
\dot{\mathrm{y}}_{\mathrm{n}+1}-\dot{\mathrm{y}}_{\mathrm{n}}=\Delta \dot{\mathrm{y}}_{\mathrm{n}}
$$

Le développement de Taylor à l'ordre 2 de l'équation (4) donne

$$
\begin{aligned}
& \mathrm{f}\left(\dot{\mathrm{x}}_{\mathrm{eq}}+\dot{\mathrm{y}}_{\mathrm{n}}, \Delta \mathrm{x}_{\mathrm{eq}}+\Delta \mathrm{y}_{\mathrm{n}}, 0+\Delta \dot{\mathrm{y}}_{\mathrm{n}}\right)=\ddot{\mathrm{y}}_{\mathrm{n}}= \\
& \mathrm{f}_{1} \dot{\mathrm{y}}_{\mathrm{n}}+\mathrm{f}_{2} \Delta \mathrm{y}_{\mathrm{n}}+\mathrm{f}_{3} \Delta \dot{\mathrm{y}}_{\mathrm{n}}+ \\
& \frac{1}{2}\left(\mathrm{f}_{12} \dot{\mathrm{y}}_{\mathrm{n}} \Delta \mathrm{y}_{\mathrm{n}}+\mathrm{f}_{13} \dot{\mathrm{y}}_{\mathrm{n}} \Delta \dot{\mathrm{y}}_{\mathrm{n}}+\mathrm{f}_{23} \Delta \mathrm{y}_{\mathrm{n}} \Delta \dot{\mathrm{y}}_{\mathrm{n}}+\mathrm{f}_{11} \dot{\mathrm{y}}_{\mathrm{n}}^{2}\right. \\
& \left.+\mathrm{f}_{22} \Delta \mathrm{y}_{\mathrm{n}}^{2}+\mathrm{f}_{33} \Delta \dot{\mathrm{y}}_{\mathrm{n}}^{2}\right)
\end{aligned}
$$

où $\left(f_{i}\right)_{1 \leq i \leq 3}$ et $\left(f_{i, j}\right)_{1 \leq i \leq j \leq 3}$ sont les dérivées partielles d'ordre 1 et 2 prises au point d'équilibre $\mathrm{f}\left(\dot{\mathrm{x}}_{\mathrm{eq}}, \Delta \mathrm{x}_{\mathrm{eq}}, 0\right)$ Le principe de cette étude est le suivant : la perturbation est écrite sous forme de Fourier

$$
\Delta y_{n}(t)=Y_{0} e^{i(k n-\omega t)}
$$

où $\mathrm{k}$ est le nombre d'onde $(0 \leq \mathrm{k} \leq \pi)$, $\mathrm{n}$ l'indice $\mathrm{du}$ véhicule et $\omega$ la pulsation. Pour des raisons d'écriture, on pose $z=-i \omega$.

En développement les équations (8) et (9) la relation de dispersion du système dynamique est obtenue :

$$
z^{2}-f_{1} z-f_{3} z\left(e^{i k}-1\right)=f_{2}\left(e^{i k}-1\right)
$$

Le système dynamique est dit stable si $\forall \mathrm{k} \in[0 . \pi], \operatorname{Re}(\mathrm{z})<$ 0 , c'est-à-dire si la perturbation décroît exponentiellement dans le temps. Après résolution de l'équation de dispersion, les conditions de stabilité sur les dérivées partielles peuvent être obtenues pour les lois non-coopérative et coopérative (équations (3) et (4)) :

$$
\mathrm{f}_{1}^{2}-2 \mathrm{f}_{2}-2 \mathrm{f}_{1} \mathrm{f}_{3}>0
$$

$$
-\mathrm{f}_{1}\left(\Sigma_{\mathrm{j}=0}^{\mathrm{m}} \mathrm{ja}-\frac{1}{2}\right)+\frac{\mathrm{f}_{2}}{\mathrm{f}_{1}}+\mathrm{f}_{3}<0 .
$$

Il s'agit d'une condition nécessaire et suffisante de stabilité pour l'équation (12) et d'une condition d'instabilité pour l'équation (13). La résolution de l'équation de dispersion nécessite des calculs poussés qui sont détaillés dans Monteil [10].

On peut noter que pour des grandes longueurs d'onde (qui concentrent l'essentiel des phénomènes de propagation en trafic), un développement limité de $\mathrm{z}$ à l'ordre 2 permet d'obtenir rapidement les conditions des équations (11) et (12). La coopération augmente de fait le domaine de stabilité, c'est-à-dire qu'elle augmente les jeux de paramètres possibles pour lesquels la perturbation ne se propage pas dans le diagramme espace-temps. La figure 2 montre l'augmentation de ce domaine de stabilité.

De même, à la limite de cette zone de stabilité, il est possible de déterminer par la méthode de perturbation réductrice l'équation unique d'un soliton, qui est en fait l'équation de l'onde de perturbation près de la frontière d'instabilité. Un changement de variable appropriée à partir de l'équation (9) permet de retomber sur des équations de type Korteweg-de-Vries (Kdv) dont la méthode de résolution est connue. La démarche et les détails de calcul sont explicités en détail Par Monteil et Billot qui généralise
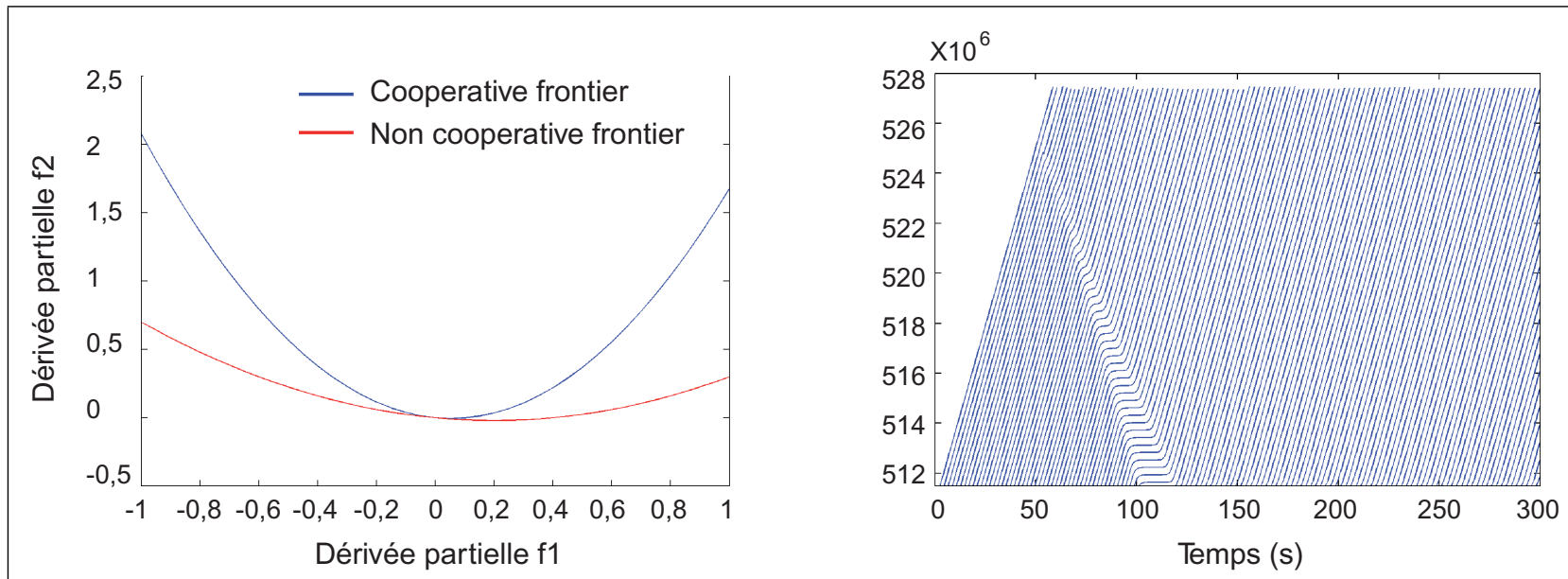

Fig. 2 (a). Domaines de stabilité (le système est stable dans la partie supérieure aux courbes). (b). Propagation d'une perturbation de type freinage (régime instable) 
et complète des travaux antérieurs. Les conclusions qui en découlent sont les suivantes : la coopération introduite diminue l'amplitude de l'équation du soliton, d'un facteur qui dépend des conditions de circulation et du modèle choisi. La vitesse de propagation de l'onde se trouve aussi augmentée, du fait de l'anticipation introduite [6] [11] [12] [13] [14] [15].

À titre d'illustration, la figure 2 montre la propagation d'une onde de choc en régime instable. Les courbes correspondant au seuil de stabilité des équations (12) et (13) sont visibles sur la figure 2 (a), pour des valeurs réalistes de $f_{1}$ et $f_{2}$, et pour $f_{2}=0,27$ (valeur obtenue après calibrage). Il est clairement visible que la coopération permet d'augmenter le domaine de stabilité, qui est la partie supérieure aux courbes. Dans le domaine instable, la perturbation se propagera, comme le montre la figure 2 (b).

\section{Simulation : calibrage et modèle OVRV-Mobil}

Le modèle microscopique servant de cadre à la simulation est la combinaison d'un modèle de poursuite (dont la forme générique a été présentée section 2 , équations (3) et (4)) et d'un modèle de changement de voie. L'objectif est d'évaluer l'impact de la stratégie coopérative sur un trafic microscopique. Un calibrage de ces modèles est ensuite réalisé dans des conditions de trafic dense. L'impact de la stratégie coopérative sur plusieurs indicateurs évaluant l'état microscopique du trafic est enfin discuté.

\section{Présentation des modèles}

Le modèle de poursuite est le modèle OVRV qui s'écrit, dans le cas multi-anticipatif [16]

$$
\ddot{x}_{n}=-\frac{1}{\tau} \dot{\mathrm{x}}_{\mathrm{n}}+\frac{1}{\tau} \mathrm{V}\left(\Delta \mathrm{x}_{\mathrm{n}}\right)+\frac{\eta}{\tau} \cdot \Delta \dot{\mathrm{x}}_{\mathrm{n}}
$$

où $\tau$ est le temps de réaction du conducteur, $\eta$ un coefficient de sensibilité du conducteur à la vitesse relative et $\mathrm{V}$ une fonction non linéaire de la distance inter-véhiculaire, définie telle que

$$
\mathrm{V}\left(\Delta \mathrm{x}_{\mathrm{n}}\right)=\frac{\mathrm{V}_{\max }}{2}\left(\tanh \left(\mathrm{s}_{\mathrm{c}}\right)+\tanh \left(\mathrm{s} .\left(\Delta \mathrm{x}_{\mathrm{n}}-\mathrm{h}_{\mathrm{c}}\right)\right)\right)
$$

$\mathrm{V}_{\max }$ étant la vitesse maximum à atteindre sur la section, $\mathrm{s}$ un coefficient de lissage et $\mathrm{h}_{\mathrm{c}}$ une distance de contrainte. Plus la distance inter-véhiculaire est proche de $\mathrm{h}_{\mathrm{c}}$, plus le véhicule aura un comportement contraint par son leader. Sous sa forme multi-anticipative, le modèle se décompose en la somme d'un terme de potentiel (fonction) et d'un terme consensuel (somme des vitesses relatives pondérées). De plus, on peut noter que, par analogie avec l'équation (9), les dérivées partielles s'écrivent : $f_{1}=-\frac{1}{\tau}, f_{2}=\frac{V^{\prime}\left(\Delta x_{\mathrm{eq}}\right)}{\tau}$ et $\mathrm{f}_{3}=\frac{\eta}{\tau}$, ce qui permettra de vérifier le critère de stabilité (12) ou (13).

Le modèle de changement de voie est le modèle Mobil qui s'écrit [17] :

$$
\begin{gathered}
\overline{\tilde{\mathrm{x}}}_{\mathrm{c}}-\ddot{\mathrm{x}}_{\mathrm{c}}+\mathrm{p}\left(\overline{\mathrm{x}}_{\mathrm{n}}-\ddot{\mathrm{x}}_{\mathrm{n}}+\overline{\mathrm{x}}_{0}-\ddot{\mathrm{x}}_{0}\right)>\Delta \mathrm{a}_{\mathrm{th}} \\
\overline{\ddot{\mathrm{x}}}_{\mathrm{n}} \geq-\mathrm{a}_{\mathrm{saf}}
\end{gathered}
$$

Où $\overline{\ddot{x}}$ représente l'accélération potentielle d'un véhicule (s'il y a changement de voie), les indices ${ }_{c}$ et ${ }_{0}$ désignent les véhicules suivant le véhicule $n$ des voies actuelles et ciblées. $\Delta \mathrm{a}_{\mathrm{th}}$ est un critère indiquant un seuil de permissivité de changement de voie, $a_{\text {saf }}$ un seuil de tolérance de l'accélération potentielle du véhicule $\mathrm{n}$. Il faut noter qu'il est possible d'introduire une asymétrie dans la manœuvre de changement de voie avec un $\Delta \mathrm{a}_{\mathrm{th} \text {,bias }}$ différent de $\Delta \mathrm{a}_{\mathrm{th}}$ si le changement de voie est fait de la voie la plus rapide à la voie la moins rapide. Enfin, p est un facteur de courtoisie, variant de à 0 à 1 pour des comportements altruistes et étant négatif pour des comportements égoïstes voire agressifs adoptés au détriment de l'efficacité et/ou de la sécurité du trafic. Comme expliqué dans (Kesting, 2007), le facteur de courtoisie est utilisé pour mesurer l'importance de la situation des véhicules $\mathrm{c}$ et $\mathrm{o}$ aux yeux du conducteur du véhicule $n$. Un comportement égoïste lors d'un changement de voie aura comme conséquence un freinage brutal du véhicule suivant dans la voie où le changement de voie s'opère, ce qui équivaut à une perturbation.

Il a été fait état d'intéressantes propriétés en simulation et calibrage pour les deux modèles présentés. En effet, le modèle OVRV a la capacité de reproduire les ondes de type stop-and-go, au même titre que la loi de poursuite IDM développée par Helbing. De plus, même si le modèle OVRV montre une variabilité des paramètres plus importante que le modèle IDM lors du calibrage, les résultats sont tout à fait corrects et la simplicité de la loi permet une interprétation plus sûre. La loi de changement de voie reproduit la fréquence des changements de voie par rapport à la densité du trafic et a l'avantage de traiter les accélérations. Ainsi, une loi Mobil combinée avec un modèle OVRV sans la perception de vitesses relatives $(\eta=0$, ce qui devient alors le modèle OVM), devient un simple modèle d'insertion de véhicules en fonction de l'espace disponible (gap acceptance) [3] [4] [18]. 
À propos de la loi coopérative, et pour la suite de la simulation, les coefficients suivants ont été choisis :

$$
\begin{gathered}
\mathrm{a}_{\mathrm{j}, \mathrm{n}}=\frac{1}{2}\left(1+\cos \left(\pi\left(\frac{\mathrm{x}_{\mathrm{j}}-\mathrm{x}_{\mathrm{n}}}{r}\right)\right)\right) \operatorname{sij} \in \mathrm{N}_{\mathrm{n}} \\
\mathrm{a}_{\mathrm{j}, \mathrm{n}}=0
\end{gathered}
$$

où $\mathrm{j} \mathrm{N}_{\mathrm{n}}, \mathrm{r}$ est le rayon de communication, égal dans notre simulation à $250 \mathrm{~m}$ car correspondant aux communications dédiées à courte portée (Jordan, 2001). Les coefficients sont normalisés. Le choix des coefficients est repris des travaux de Olfati-Saber et Saber et Murray [19] [20].

\section{Calibrage des données NGSIM}

Comme la communication inter-véhiculaire et/ou avec l'infrastructure opère à un niveau microscopique, il est souhaitable de calibrer ces modèles afin de voir dans quelles conditions réalistes une stratégie dite coopérative améliorerait l'état du trafic.

Pour cela, nous avons utilisé les données de trajectoires des deux voies les plus rapides de la section de l'autoroute US 101 à Los Angeles, collectées le 15 juin 2005. Ces données font partie des données NGSIM largement usitées dans la communauté [21].

La section était composée de 5 voies avec une entrée et une sortie d'autoroute. Nous nous sommes concentrés sur les deux voies les plus rapides, car nous étudions les phénomènes de comportement en poursuite ainsi que les changements de voie et ne voulions pas être influencés par les entrées et sorties. La voie rapide est notée voie 1, l'autre voie 2.

Pour le processus de calibrage détaillé, nous renvoyons le lecteur à Monteil et al. et aux travaux de Kesting [18] [22].

Un filtrage des données était nécessaire préalablement au calibrage. Des filtrages successifs de type EMA (moyenne glissante exponentielle) ont été effectués sur les positions, vitesses puis accélérations. Ensuite, la fonction objective est choisie telle que

$$
E=\sqrt{\frac{1}{\left\langle s_{\text {data }}\right\rangle}\left\langle\frac{\left(s_{\text {sim }}-s_{\text {data }}\right)^{2}}{s_{\text {data }}}\right\rangle}
$$

où $\mathrm{s}_{\text {sim }}$ et $\mathrm{s}_{\text {data }}$ sont une mesure des trajectoires simulées et observées et où est leur moyenne temporelle sur toute la simulation.et sont définies telles que $\mathrm{s}=\mathrm{x}+0,5 * \dot{\mathrm{x}}$, ceci reportant de meilleurs résultats en simulation [23].

Des pénalités sont ajoutées lors du calibrage pour empêcher des accélérations $\left(3,5 \mathrm{~m} / \mathrm{s}^{2}\right)$ et décélérations trop fortes $\left(-7,5 \mathrm{~m} / \mathrm{s}^{2}\right)$. Une optimisation par algorithme génétique donne ensuite le vecteur des paramètres optimaux sous la forme :

$$
\left(\mathrm{V}_{\max }, \tau, \eta, \mathrm{h}_{\mathrm{c}}, \mathrm{s}\right)
$$

Les résultats donnent pour les voies 1 et 2 combinées (30,9, $1,98,0,54,45,3,0,28)$. Le trafic dense, les différences de comportement entre les deux voies sont minimes et ne sont pas le sujet de l'étude. Les unités ont été conservées en feet $/ \mathrm{s}$, en secondes (s) et en feet.

Le calibrage du modèle de poursuite effectué, nous nous intéressons au modèle de changement de voie. Pour cela une distribution des vitesses maximales est utilisée de telle sorte que $\tilde{\mathrm{V}}_{\max } \sim \mathcal{N}\left(\mathrm{V}_{\max }, 2\right)$. Cette approximation nous est pertinente dans la mesure où l'objectif premier n'est pas de reproduire le trafic, mais d'obtenir des paramètres réalistes mettant en jeu le comportement des conducteurs. Cette fois la fonction objective est fondée sur la fréquence des changements de voie, une nouvelle optimisation par algorithme génétique de $\left(\mathrm{a}_{\mathrm{saf}}, \mathrm{p}, \Delta \mathrm{a}_{\mathrm{th}}, \Delta \mathrm{a}_{\mathrm{th}, \text { bias }}\right)$ conduit aux paramètres $(8,0,5,0,9,1,2)$, avec comme unité d'accélération le feet $/ \mathrm{s}^{2}$.

\section{Indicateurs de stabilité/performance : que cherche-t-on à améliorer ?}

Il est question d'évaluer l'effet de la loi coopérative longitudinale sur le trafic microscopique, dans un premier temps sur les comportements de type longitudinal mais aussi son influence sur les mouvements de type latéral. Pour cela une définition d'indicateurs appropriés et pertinents est nécessaire. Ces indicateurs doivent être des indicateurs microscopiques, par ex. qui mesurent l'état local du voisinage de chaque véhicule.

Nous introduisons un indicateur dit de désordre du groupe, censé mesurer l'entropie des vitesses à chaque pas de temps. Il a été introduit dans Choi et al. de la manière suivante [24] :

$$
\operatorname{DG}(\mathrm{v})=\frac{1}{4} \Sigma_{(\mathrm{i}, \mathrm{j}) \in \psi(\mathrm{v})} \mathrm{a}_{\mathrm{i}, \mathrm{j}}\left|\mathrm{v}_{\mathrm{j}}-\mathrm{v}_{\mathrm{i}}\right|
$$

Où $\Psi(\mathrm{p})$ est l'ensemble défini tel que $(\mathrm{i}, \mathrm{j}) \quad \Psi(\mathrm{p})$ si et seulement si les véhicules $i$ et $j$ peuvent communiquer entre eux, c'est-à-dire qu'ils sont séparés de moins de $250 \mathrm{~m}$ sur une même voie.

Les autres indicateurs sont bien connus : la distribution des vitesses, la distribution du temps de parcours, la distribution des distances inter-véhiculaires, le nombre de changement de lignes, et enfin la distribution des temps avant collision (time to collision, TTC). Le temps avant collision est défini comme le temps requis pour que deux 
véhicules rentrent en collision s'ils gardent la même vitesse, soit le rapport entre distance inter-véhiculaire et vitesse relative [25].

\section{Effets de la stratégie coopérative}

Des simulations d'une durée de 8 minutes ont été réalisées pour les deux voies. Les paramètres précédemment calibrés sont utilisés. Une distribution des vitesses maximum a été choisie égale à : $\tilde{\mathrm{V}}_{\max } \sim \mathcal{N}\left(\mathrm{V}_{\max }, 2\right)$, de manière à créer une hétérogénéité et à favoriser les changements de voie. Le flux entrant est de $0,49 \mathrm{veh} / \mathrm{s}$, choisi comme moyenne des données empiriques.

La figure 3 montre une simulation sans ajout de perturbation (ni freinage ni changement de voie intempestif). Dans ce cas, si la loi permet d'homogénéiser le trafic, elle provoque également une diminution de la moyenne des vitesses et donc une augmentation du temps de parcours.

Les résultats sont à mettre en relief avec les résultats de la section 3 sur les critères de stabilité. Si le critère de stabilité (équation (12)) n'est pas respecté, on est en régime instable. Ici, les valeurs des dérivées partielles $f_{1}=-0,51$, $\mathrm{f}_{2}=0,05$ et $\mathrm{f}_{3}=0,27$ indiquent un régime stable d'après l'équation (12). Ceci n'a rien d'incompatible avec un régime dense (véhicules évoluant à $35 \mathrm{~km} / \mathrm{h}$ sur autoroute), qui est la situation de trafic considérée dans l'échantillon de données.

Plaçons-nous dans le cas dans le cas où $30 \%$ des véhicules ont un comportement de changement de voie égoïste $(\mathrm{p}=-1)$. Ce type de comportement peut provoquer une perturbation spontanée, suite à une manœuvre de changement de voie intempestive. Si le trafic est

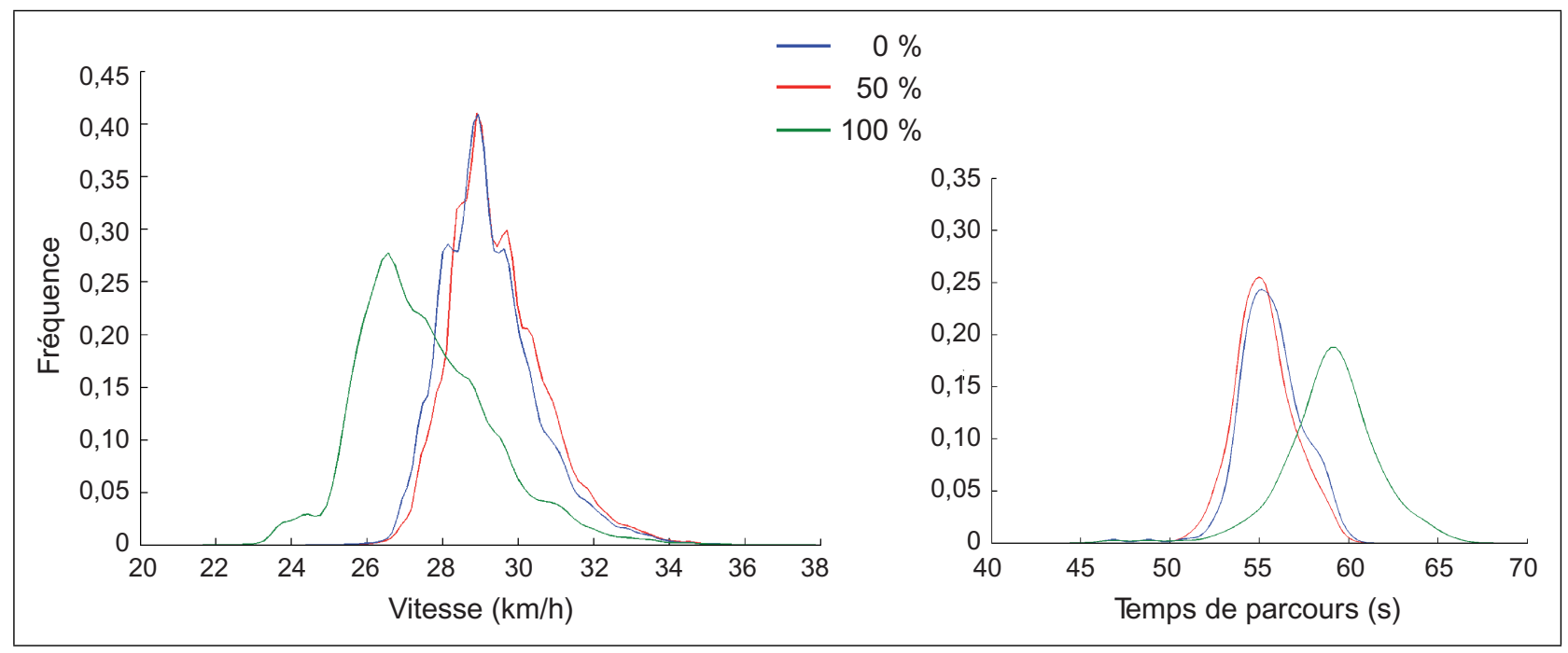

Fig. 3 Distribution des vitesses et temps de parcours sans perturbations, pour $0 \%, 50 \%$ et $100 \%$ de véhicules équipés

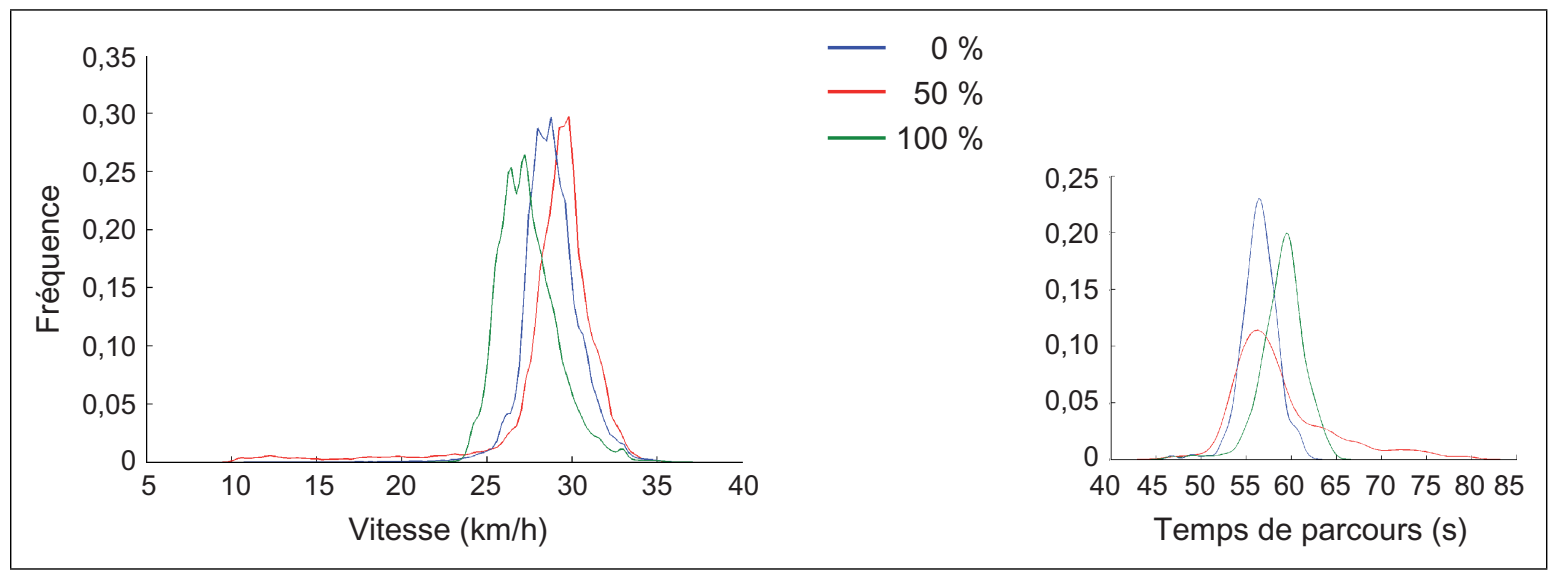

Fig. 4 Distribution des vitesses et temps de parcours avec $30 \%$ de comportement agressif de changement de voie, pour $0 \%$, $50 \%$ et $100 \%$ de véhicules équipés 


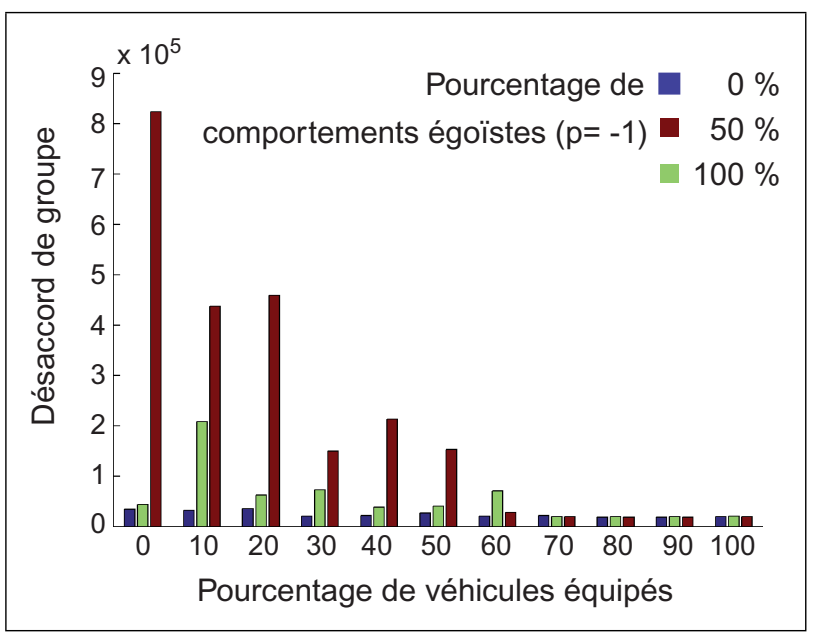

Fig. 5 Indicateur de désordre de groupe : impact du pourcentage de comportements égoïstes et de véhicules équipés

globalement dans un régime stable, on observe que des perturbations se propagent avant de progressivement se résorber. Nous pensons que ceci est dû à un état de trafic localement instable généré par les comportements agressifs et réalisés au détriment des véhicules suivants. La figure 4, qui montre les distributions de vitesse et de temps de parcours dans cette situation, témoigne de ce phénomène. La courbe pour la loi non-coopérative (courbe en rouge) montre que certaines vitesses sont proches de 0 , et il s'agit clairement de congestion de type stop and go. De plus, la distribution du temps de parcours s'étale jusqu'à plus de 80 secondes. Il est aussi visible que dans cette situation de trafic, $50 \%$ de véhicules équipés est un pourcentage suffisant pour avoir un effet stabilisant, c'est-à-dire effacer les situations localement instables. À partir d'un certain pourcentage d'équipement, l'effet stabilisant est suffisant, il n'est donc pas nécessaire d'augmenter ce pourcentage (et cela a de nouveau un effet négatif sur le temps de parcours, voir figure 4). Cette assertion est vérifiée figure 5.

En effet, l'impact de cette loi de poursuite coopérative sur les manœuvres de changement de voie est étudié. La figure 5 caractérise le désordre de groupe en fonction du pourcentage de véhicules équipés et de véhicules à comportement égoïste. Il est visible que la loi coopérative gomme l'effet de ces comportements égoïstes. Ceci s'explique par l'effet stabilisant de la loi multi-anticipative. L'homogénéisation opérée par la loi multi-anticipative tend à supprimer le phénomène d'instabilités locales, que nous croyons être à l'origine de l'augmentation du temps de parcours. Le seuil pour lequel les comportements agressifs n'ont plus aucun impact sur le désaccord de groupe se situe entre $60 \%$ et $70 \%$. En ce sens la figure 5 corrobore l'intuition faisant suite aux résultats des figures 3 et 4 .

En plus de ces indicateurs d'efficacité et de stabilisation, l'effet de la coopération sur la sécurité du trafic est à présent évalué. La figure 6 atteste des distributions de deux indicateurs de sécurité, la distance inter-véhiculaire et le temps avant collision. Si la coopération a tendance à réduire la distance et le temps inter-véhiculaire, elle a un effet très positif sur le temps avant collision. En effet, un véhicule qui prend en compte l'information de multiples leaders réagira de façon moins sévère à son leader, et donc la distance et le temps inter-véhiculaire se trouve réduits. Mais en ce qui concerne la sécurité, la loi multi-anticipative a un effet très positif sur le temps avant collision, ce qui est dû au rôle homogénéisant de la loi multi-anticipative, notamment au terme de consensus dans la loi de poursuite (équation (14)).

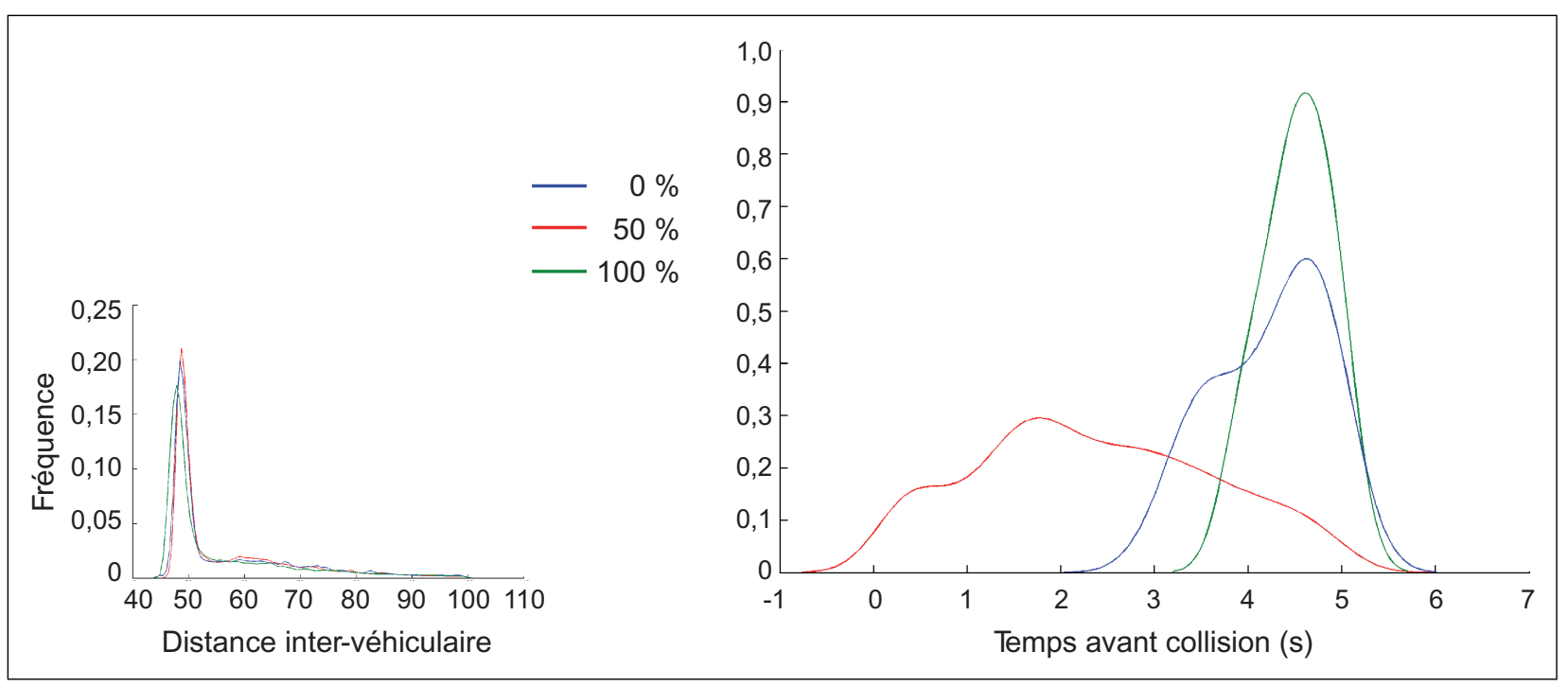

Fig. 6 Indicateurs de sécurité. Distribution (a). Des distances inter-véhiculaires (b). Des temps avant collisions 
Pour conclure ces observations, il convient de rappeler une limite à ce travail. Il est en effet probable qu'une flotte mixte de véhicules (équipés et non-équipés) aura un effet sur les paramètres des modèles microscopiques. Les conducteurs seraient susceptibles de réagir différemment s'ils observent un comportement différent des conducteurs recevant des consignes, par ex. obéissant à la loi multianticipative.

\section{Discussion et conclusions}

Les travaux présentés se sont concentrés sur l'intégration des systèmes coopératifs dans les modèles microscopiques de trafic. En postulant que la communication se traduit d'abord en une anticipation nouvelle du conducteur, des lois de poursuite multi-anticipatives ont été appliquées aux formes génériques de modèles microscopiques.

Des calculs et analyses de stabilité permettent de conclure sur les possibles avantages et limites de cette anticipation. L'étude analytique fait état de deux améliorations notables, en terme de domaine de stabilité d'une part et en terme de structure des ondes de chocs d'autre part. Il en résulte un trafic plus stable donc moins sujet à la propagation des perturbations et la congestion qui peut en résulter. Du fait d'une réduction de l'amplitude de l'onde de choc, la loi multi-anticipative se traduira par une réduction du temps perdu. Cependant, les résultats montrent qu'une telle stratégie doit être adaptée aux conditions de trafic, car si elle a toujours tendance à homogénéiser le trafic par une harmonisation des vitesses, elle peut aussi augmenter les temps de parcours. L'impact sur des indicateurs de sécurité comme le temps avant collision montre à quel point une communication entre véhicules sécurise le flot de véhicules. En cas de déploiement massif des systèmes coopératifs, et dans une perspective de gestion de trafic temps réel, un compromis entre sécurité et efficacité devra être trouvé. Il consistera à utiliser la communication entre véhicules de manière adaptée aux conditions de trafic. En parallèle d'un modèle de trafic décrivant la dynamique des véhicules, une stratégie de changement de voie a été introduite. Cette stratégie repose sur des facteurs de courtoisie et des comportements plus ou moins altruistes. Les résultats des simulations montrent que la communication entre véhicules gomme les comportements égoïstes/ abusifs des conducteurs. L'effet stabilisant-homogénéisant, qui était intuitif, a donc pu être vérifié de manière analytique par des études de stabilité, et par simulation par la comparaison d'indicateurs d'efficacité et de sécurité. Les limites de cette étude sont liées aux hypothèses sur les modèles et le comportement des conducteurs supposé quasi-déterministe. En effet, les seules sources d'incertitude considérées sont les distributions des vitesses maximums, le pourcentage de conducteurs égoïstes pour le changement de voie et le pourcentage de véhicules coopératifs. Mais il y a d'autres incertitudes qui appellent à un besoin de modélisation. En particulier, nos premiers travaux supposent une adhésion totale des conducteurs à l'information qu'ils reçoivent voire un comportement quasi-automatisé des véhicules. La question de la perception de l'information et des comportements qui en découlent doit être traitée à la lumière de travaux menés en psychologie du conducteur. Au niveau de la communication, des biais dus aux temps de latence ou autre interférence dans la communication doivent être incorporés à notre démarche. Aussi, les données transmises ne sont pas toujours exactes car estimées. Enfin, l'information peut être transmise au sein d'un réseau faisant apparaître une variété des schémas de communication.

Mais ce travail soulève également la limite même des approches classiques pour la modélisation des systèmes coopératifs. Si les modèles multi-anticipatifs sont un premier effort pour rendre compte d'une communication entre véhicules, l'approche reste limitée et les interactions sont bien plus complexes. Prendre en compte les communications entre l'infrastructure et les véhicules nécessite certainement d'envisager d'autres formalismes. Le système serait ici composé des différentes entités communicantes et il s'agit de modéliser à une échelle plus vaste la nature des interactions entre les différents acteurs et les lois de transition entre les états du système. Les systèmes multiagents, les réseaux bayésiens ou des approches inspirées des automates à états finis pourraient être envisagés pour satisfaire ces objectifs [19] [26].

Enfin, du point de vue d'une gestion coopérative du trafic, il faut aussi bien s'interroger sur la nature des informations à envoyer aux conducteurs que sur les critères à optimiser. Nous avons proposé ici des critères d'homogénéisation ou de stabilisation du trafic qui doivent s'inscrire à l'avenir dans une optimisation multi-objectif et temps réel du réseau, sensibles aux conditions locales du trafic. En conclusion, nous pouvons dire que le chemin vers une route intelligente et automatisée est encore long. En cas d'une industrialisation massive des systèmes coopératifs, la phase transitoire d'équipement progressif de la flotte de véhicules et des infrastructures autant que la multiplication des informations transmissibles soulèveront de nombreux défis pour les chercheurs et opérateurs qui se trouveront confrontés à une forme ultime de gestion du trafic. 


\section{Références}

1. Shladover SE et al. (2012) Impact of cooperative cruise control on freeway traffic flow, Proceedings of the 2012 annual meeting of the Transportation Research Board, TRB, Washington DC.

2. Ngoduy D, Hoogendoorn SP, Liu R (2009) Continuum modeling of cooperative traffic flow dynamics. Physica A: Statistical Mechanics and its Applications 388(13): 2705-2716.

3. Bando M, Hasebe K, Nakayama A, Shibata A, Sugiyama Y (1995) Dynamical model of traffic congestion and numerical simulation. Phys. Rev. E 51(2): 1035-1042.

4. Helbing D, Tilch B (1998) Generalized force model of traffic dynamics. Phys. Rev. E 58(1): 133-138.

5. Treiber M, Hennecke A, Helbing D (2000) Congested traffic states in empirical observations and microscopic simulations, Physical Review E 62(2): 1805-1824, doi:10.1103/PhysRevE.62.1805

6. Ge H, Dai XSQ, Dong LY (2006) An extended car-following model based on intelligent transportation system application. Physica A: Statistical Mechanics and its Applications 365(2): 543-548.

7. Ioannou P, Bose A (1999) Automated Vehicle Control Handbook of Transportation Science, Ed. R. Hall, Kluwer Academic Publishers, pp.187-233.

8. Treiber M, Kesting A (2011) Evidence of convective instability in congested traffic flow: A systematic empirical and theoretical investigation. 19th International Symposium on Transportation and Traffic Theory Procedia - Social and Behavioral Sciences, Volume 17, pp. 683-701.

9. Kerner B (2012) Phys. Rev. E 85, 036110.

10. Monteil J, Billot R, El Faouzi NE (2012) Distributed and centralized approaches for cooperative road traffic. Procedia Social and Behavioral Sciences, 48:3198, 3208, 2012.

11. Saadi F, Azizpour J, Zahedi SA (2012) Analytical solutions of Korteweg De Vries Equation. World academy of science, engineering and technology 69.

12. Drazin PG, Johnson RS (1989) Solitons: an introduction / P.G. Drazin, R.S. Johnson (Cambridge University Press, Cambridge [England] ; New York) pp. xii, $226 \mathrm{p}$.

13. Monteil J, Billot R (2012) Techniques de stabilité pour la modélisation des effets des systèmes cooperatifs sur le trafic. Rapport technique, LICIT, ENTPE-IFSTTAR.

14. Nagatani $T$ (1998) Thermodynamic theory for the jamming transition in traffic flow. Physical Review E (Statistical Physics, Plasmas, Fluids, and Related Interdisciplinary Topics) 58(4): 4271-4276

15. Masakuni M, Nagatani T (1999) Soliton and kink jams in traffic flow with open boundaries [J]. Phys Rev E 60(1): 180-187.

16. Jiang J, Wu Q, Zhu Z (2001) Full velocity difference model for a car-following theory Phys. Rev. E 64, 017101.

17. Kesting A, Treiber M, Helbing D (2007) General Lane-Changing Model MOBIL for Car-Following Models. Transportation Research Record 1999: 86-94.

18. Kesting A, Treiber M (2008) Calibrating Car-Following Models by using Trajectory Data: Methodological Study. Transportation Research Record 2088: 148-156. DOI: 10.3141/2088-16

19. Olfati-Saber R (2006) Flocking for multi-agent dynamic systems: Algorithms and theory. IEEE Transactions on Automatic Control 51:401-420.
20. Saber RO, Murray RM (2003) Flocking with obstacle avoidance: cooperation with limited communication in mobile networks. Decision and Control. Proceedings. 42nd IEEE Conference on, vol. 2, pp. 2022-2028

21. Punzo V, Borzacchiello M, Ciuffo B (2011) On the assessment of vehicle trajectory data accuracy and application to the Next Generation SIMulation (NGSIM) program data, Transportation Research Part C: Emerging Technologies 19(6): 1243-1262.

22. Monteil J, Nantes A, Billot R, El Faouzi NE (2012) Microscopic cooperative traffic based on NGSIM data. Proceedings of the 19th ITS World Congress, Vienna, 2012.

23. Bevrani K, Chung E (2011) Car following model improvement for traffic safety metrics reproduction. Proceedings of the Australasian Transport Research Forum 2011, PATREC, Adelaide Hilton Hotel, Adelaid, SA, pp. 1-14. (In Press).

24. Choi J, Oh S, Horowitz R (2009) Distributed learning and cooperative control for multi-agent systems. Automatica 45: 2802-2814

25. Hayward JC (1972). Near miss determination through use of a scale of danger. Report no. TTSC 7115, The Pennsylvania State University, Pennsylvania.

26. Armetta F, Hassas S,Pimont S, Lefevre O (2006) Towards the Control of Emergence by the Coordination of Decentralized Agent Activity for the Resource Sharing Problem. Engineering Self-Organising Systems, 4th International Workshop: $132-150$

\section{Références complémentaires}

Car 2 Car communication consortium (2007) CAR 2 CAR Communication Consortium Manifesto-Overview of the $\mathrm{C} 2 \mathrm{C}-\mathrm{CC}$ System.

Ge HX, Dai SQ, Xue Y, Dong LY (2005) Stabilization analysis and modified korteweg-de vriesequation in a cooperative driving system. Phys. Rev. E 71(6): 066119.

Helbing D (2001) Traffic and related self-driven many-particle systems. Rev. Mod. Phys. 73(4):1067-275 1141.

Jordán JG, Soriano F, Graullera D, Martín G (2001) A comparison of different technologies for EFC and other ITS applications Proceedings of the IEEE Conference on Intelligent Transportation Systems (ITSC '01), pp. 1171-1176, Oakland, USA.

Monteil J. Billot R, El Faouzi NE (2011) Towards cooperative traffic management: methodological issues and perspectives. Australasian Transport Research Forum 2011 Proceedings, Adelaide, Australia

Schonhof M, Treiber M, Kesting A, Helbing D (2007) Autonomous detection and anticipation of jam fronts from messages propagated by inter-vehicle communication. Transportation research record 1999(3).

Wilson EJ, Ward A (2011) Car-following models: fifty years of linear stability analysis - a mathematical perspective, Transportation Planning and Technology $34: 3-18$.

Treiber M, Kesting A (2013) Traffic Flow Dynamics. Springer.

Yahya B, Ben-Othman J (2009) Towards a classification of energy aware MAC protocols for wireless sensor networks, Wireless Communications and Mobile Computing 9(12) : 1572-1607. 